\title{
Permutation-information-theory approach to unveil delay dynamics from time-series analysis
}

\author{
L. Zunino, ${ }^{1,2,3, *}$ M. C. Soriano, ${ }^{1, \dagger}$ I. Fischer, ${ }^{1, \ddagger}$ O. A. Rosso, ${ }^{4,5, \S}$ and C. R. Mirasso ${ }^{1, \|}$ \\ ${ }^{1}$ Instituto de Física Interdisciplinar y Sistemas Complejos (IFISC), CSIC-UIB, Campus Universitat de les Illes Balears, \\ E-07122 Palma de Mallorca, Spain \\ ${ }^{2}$ Centro de Investigaciones Ópticas (CONICET La Plata-CIC), C.C. 3, 1897 Gonnet, Argentina \\ ${ }^{3}$ Departamento de Ciencias Básicas, Facultad de Ingeniería, Universidad Nacional de La Plata (UNLP), 1900 La Plata, Argentina \\ ${ }^{4}$ Instituto de Ciências Exatas (Fisica), Universidade Federal de Minas Gerais, Av. Antônio Carlos 6627, Campus Pampulha, \\ Belo Horizonte, MG 31270-901, Brazil \\ ${ }^{5}$ Chaos \& Biology Group, Instituto de Cálculo, Facultad de Ciencias Exactas y Naturales, Universidad de Buenos Aires, \\ Pabellón II, Ciudad Universitaria, 1428 Ciudad de Buenos Aires, Argentina
}

(Received 21 May 2010; revised manuscript received 9 August 2010; published 18 October 2010)

\begin{abstract}
In this paper an approach to identify delay phenomena from time series is developed. We show that it is possible to perform a reliable time delay identification by using quantifiers derived from information theory, more precisely, permutation entropy and permutation statistical complexity. These quantifiers show clear extrema when the embedding delay $\tau$ of the symbolic reconstruction matches the characteristic time delay $\tau_{S}$ of the system. Numerical data originating from a time delay system based on the well-known Mackey-Glass equations operating in the chaotic regime were used as test beds. We show that our method is straightforward to apply and robust to additive observational and dynamical noise. Moreover, we find that the identification of the time delay is even more efficient in a noise environment. Our permutation approach is also able to recover the time delay in systems with low feedback rate or high nonlinearity.
\end{abstract}

DOI: 10.1103/PhysRevE.82.046212

PACS number(s): 05.45.Tp, 89.70.Cf, 02.30.Ks

\section{INTRODUCTION}

When studying dynamical phenomena in nature the corresponding underlying equations or even the relevant governing mechanisms are often not known. In fact the starting point to study many of these systems is a set of measurements of some representative variable of interest at discrete time intervals, i.e., a black box time series, given by the set $S=\left\{x_{t}, t=1, \ldots, N\right\}$, with $N$ being the number of observations. An important problem in the analysis of time-series data is the identification of delayed feedback or delayed interaction mechanisms present in the dynamics since delay phenomena are intrinsic to many dynamical processes. The identified delay can give information about the interaction between the system components. It is then necessary to discriminate the presence of time delays in order to develop suitable models for simulation and forecasting purposes. Time delayed dynamics are naturally required and implemented to model many real systems in different fields including biology [1-3], optics [4-6], and climatology [7], among others. Therefore, the identification from a time series of a possible delay present in the system has become one of the key problems in the study of nonlinear dynamical systems.

Numerous approaches were previously proposed to determine the unknown delay time $\tau_{S}$ from recorded time series. Conventional and widely applied tools are the autocorrelation function [8] and the delayed mutual information (DMI)

\footnotetext{
*lucianoz@ciop.unlp.edu.ar

†miguel@ifisc.uib-csic.es

†ingo@ifisc.uib-csic.es

§oarosso@ fibertel.com.ar

"claudio@ifisc.uib-csic.es
}

$[9,10]$. More recently new techniques were introduced. Without being exhaustive we can mention the minimal forecast error $[11,12]$, several methods from information theory $[13,14]$, the filling factor analysis introduced by Bünner $e t$ al. [15], the statistical analysis of time intervals between extrema in the time series [16], and the practical criterion recently proposed by Siefert [17]. In this paper we introduce an approach by using quantifiers derived from information theory, more precisely entropy and statistical complexity. It should be stressed that, in order to evaluate these quantifiers, a particularly efficient symbolic technique, the Bandt and Pompe permutation method [18], is used to estimate the probability distribution associated with the time series. As it is widely known, symbolic time-series analysis methods that discretize the raw time series into a corresponding sequence of symbols have the potential of analyzing nonlinear data efficiently with low sensitivity to noise [19]. However, finding a meaningful symbolic representation of the original series is not an easy task [20,21]. The Bandt and Pompe approach is the only symbolization technique among those in popular use that takes into account time causality of the system's dynamics. Then, important details concerning the ordinal structure of the time series are revealed [22-31].

As will be discussed in detail below, we have found that the permutation entropy is minimized and the permutation statistical complexity is maximized when the embedding delay $\tau$ of the symbolic reconstruction matches the intrinsic time delay $\tau_{S}$ of the system. The reliability of our methodology is tested using numerical time series obtained from the widely used Mackey-Glass equation subject to a time delay, operating in a chaotic regime. The main advantages of our quantifiers are their simplicity and robustness to noise. Most importantly, we have found a resonancelike behavior in the presence of observational and dynamical noise; i.e., the iden- 
tification of the time delay is improved in a noise environment.

\section{PERMUTATION ENTROPY AND PERMUTATION STATISTICAL COMPLEXITY}

The information content of a system is typically evaluated from a probability distribution $P$ describing the distribution of some measurable or observable quantity. An information measure can primarily be viewed as a quantity that characterizes this given probability distribution. Shannon entropy is very often used as a first natural approach. Given any arbitrary probability distribution $P=\left\{p_{i}: i=1, \ldots, M\right\}$, the widely known Shannon's logarithmic information measure, $S[P]$ $=-\sum_{i=1}^{M} p_{i} \ln p_{i}$, is regarded as the measure of the uncertainty associated with the physical process described by $P$. If $S[P]=0$ we are in position to predict with complete certainty which of the possible outcomes $i$ whose probabilities are given by $p_{i}$ will actually take place. Our knowledge of the underlying process described by the probability distribution is maximal in this instance. In contrast, our knowledge is minimal for a uniform distribution.

It is widely known that an entropy measure does not quantify the degree of structure or patterns present in a process [32]. Moreover, it was recently shown that measures of statistical or structural complexity are necessary because they capture the property of organization [33]. This kind of information is not discriminated by randomness measures. The opposite extremes of perfect order and maximal randomness (a periodic sequence and a fair coin toss, for example) possess no complex structure, and then these systems are too simple and should have zero statistical complexity. At a given distance from these extremes, a wide range of possible degrees of physical structure exists, which should be quantified by the statistical complexity measure (SCM). Lamberti et al. [34] introduced an effective SCM that is able to detect essential details of the dynamics and differentiate different degrees of periodicity and chaos. It provides important additional information regarding the peculiarities of the underlying probability distribution, not already detected by the entropy. This statistical complexity measure is defined, following the intuitive notion advanced by López-Ruiz et al. [35], through the product

$$
\mathcal{C}_{J S}[P]=\mathcal{Q}_{J}\left[P, P_{e}\right] \mathcal{H}_{S}[P]
$$

of the normalized Shannon entropy

$$
\mathcal{H}_{S}[P]=S[P] / S_{\max },
$$

with $\quad S_{\max }=S\left[P_{e}\right]=\ln M \quad\left(0 \leq \mathcal{H}_{S} \leq 1\right) \quad$ and $\quad P_{e}$ $=\{1 / M, \ldots, 1 / M\}$ as the uniform distribution, and the disequilibrium $\mathcal{Q}_{J}$ defined in terms of the extensive (in the thermodynamical sense) Jensen-Shannon divergence. That is, $\mathcal{Q}_{J}\left[P, P_{e}\right]=\mathcal{Q}_{0} \mathcal{J}\left[P, P_{e}\right] \quad$ with $\mathcal{J}\left[P, P_{e}\right]=\left\{S\left[\left(P+P_{e}\right) / 2\right]\right.$ $\left.-S[P] / 2-S\left[P_{e}\right] / 2\right\}$ the above-mentioned Jensen-Shannon divergence and $\mathcal{Q}_{0}$ a normalization constant equal the inverse of the maximum possible value of $\mathcal{J}\left[P, P_{e}\right]$. This value is obtained when one of the components of $P$, say $p_{m}$, is equal to 1 and the remaining $p_{i}$ are equal to zero. The Jensen-
Shannon divergence, which quantifies the difference between two (or more) probability distributions, is especially useful to compare the symbol composition between different sequences [36]. The complexity measure constructed in this way is intensive, similarly to many thermodynamic quantities [34]. We stress the fact that the above SCM is not a trivial function of the entropy because it depends on two different probabilities distributions: the one associated with the system under analysis, $P$, and the uniform distribution, $P_{e}$. Furthermore, it was shown that for a given $\mathcal{H}_{S}$ value, there exists a range of possible SCM values [37,38]. Thus, it is clear that important additional information related to the correlational structure between the components of the physical system is provided by evaluating the statistical complexity [39].

In order to evaluate the two above-mentioned quantifiers, $\mathcal{H}_{S}$ and $\mathcal{C}_{J S}$, an associated probability distribution should be constructed beforehand. The adequate way of choosing the probability distribution associated with a time series is an open problem. Rosso et al. [23] recently showed that improvements in the performance of information quantifiers, such as entropy and statistical complexity measures, can be expected if the time causality of the system dynamics is taken into account when computing the underlying probability distribution. Specifically, it was found that these information measures allow us to distinguish between chaotic and stochastic dynamics when causal information is incorporated into the scheme to generate the associated probability distribution. Bandt and Pompe [18] introduced a successful method to evaluate the probability distribution considering this time causality. They suggested that the symbol sequence should arise naturally from the time series, without any model assumptions. Thus, they took partitions by comparing the order of neighboring values rather than partitioning the amplitude into different levels. That is, given a time series $\left\{x_{t}, t=1, \ldots, N\right\}$, an embedding dimension $D>1$, and an embedding delay time $\tau$, the ordinal pattern of order $D$ generated by

$$
s \mapsto\left(x_{s-(D-1) \tau}, x_{s-(D-2) \tau}, \ldots, x_{s-\tau}, x_{s}\right)
$$

has to be considered. To each time $s$ we assign a $D$-dimensional vector that results from the evaluation of the time series at times $s-(D-1) \tau, \ldots, s-\tau, s$. Clearly, the higher the value of $D$, the more information about the past is incorporated into the ensuing vectors. By the ordinal pattern of order $D$ related to the time $s$ we mean the permutation $\pi=\left(r_{0}, r_{1}, \ldots, r_{D-1}\right)$ of $(0,1, \ldots, D-1)$ defined by

$$
x_{s-r_{0} \tau} \geq x_{s-r_{1} \tau} \geq \cdots \geq x_{s-r_{D-2} \tau} \geq x_{s-r_{D-1} \tau} .
$$

In this way the vector defined by Eq. (3) is converted into a unique symbol $\pi$. The procedure can be better illustrated with a simple example; let us assume that we start with the time series $\{1,3,5,4,2,5, \ldots\}$, and we set the embedding dimension $D=4$ and the embedding delay $\tau=1$. In this case the state space is divided into 4 ! partitions and 24 mutually exclusive permutation symbols are considered. The first fourdimensional vector is $(1,3,5,4)$. According to Eq. (3) this vector corresponds to $\left(x_{s-3}, x_{s-2}, x_{s-1}, x_{s}\right)$. Following Eq. (4) 
we find that $x_{s-1} \geq x_{s} \geq x_{s-2} \geq x_{s-3}$. Then, the ordinal pattern which allows us to fulfill Eq. (4) will be $(1,0,2,3)$. The second four-dimensional vector is $(3,5,4,2)$, and $(2,1,3,0)$ will be its associated permutation, and so on. In order to get a unique result we consider that $r_{i}<r_{i-1}$ if $x_{s-r_{i}}=x_{s-r_{i-1}} \tau$. This is justified if the values of $x_{t}$ have a continuous distribution, so that equal values are very unusual. Otherwise, it is possible to break these equalities by adding small random perturbations. For all the $D$ ! possible permutations $\pi_{i}$ of order $D$, their associated relative frequencies can be naturally computed by the number of times this particular order sequence is found in the time series divided by the total number of sequences. Thus, an ordinal pattern probability distribution $P=\left\{p\left(\pi_{i}\right), i=1, \ldots, D !\right\}$ is obtained from the time series. This way of symbolizing time series, based on a comparison of consecutive points, allows a more accurate empirical reconstruction of the underlying phase space of chaotic time series affected by weak (observational and dynamical) noise [18]. To determine $p\left(\pi_{i}\right)$ exactly an infinite number of terms in the time series should be considered, i.e., $N \rightarrow \infty$ to determine the relative frequencies. This limit exists with probability 1 when the underlying stochastic process fulfills a very weak stationarity condition: for $k \leq D$, the probability for $x_{t}<x_{t+k}$ should not depend on $t$ [18]. The probability distribution $P$ is obtained once we fix the embedding dimension $D$ and the embedding delay time $\tau$. The former parameter plays an important role for the evaluation of the appropriate probability distribution since $D$ determines the number of accessible states, given by $D$ !. Moreover, it was established [40] that the length $N$ of the time series must satisfy the condition $N$ $\gg D$ ! in order to obtain a reliable statistics. With respect to the selection of the other parameter, Bandt and Pompe specifically considered an embedding delay $\tau=1$ in their cornerstone paper [18]. Nevertheless, it is clear that other values of $\tau$ could provide additional information.

In this work we evaluate the normalized Shannon entropy $\mathcal{H}_{S}$ [Eq. (2)] and the SCM $\mathcal{C}_{J S}$ [Eq. (1)] using the permutation probability distribution $P=\left\{p\left(\pi_{i}\right), i=1, \ldots, D\right.$ ! $\}$. Defined in this way, the former quantifier is called permutation entropy and the latter is called permutation statistical complexity.

\section{NUMERICAL RESULTS AND DISCUSSION}

To estimate the quantifiers, permutation entropy and permutation statistical complexity, it is necessary to fix the embedding dimension and the embedding delay. It is clear that the condition $N \gg D$ ! limits the possible values for the embedding dimension. However, a study about the influence of the embedding delay is still lacking. We hypothesize that this parameter could be strongly related, if it is relevant, with the intrinsic time delay of the system under analysis. In order to check this hypothesis we have estimated the permutation entropy and the permutation statistical complexity as functions of the embedding delay $\tau$ for the well-known Mackey-Glass equation, a paradigmatic time delay system. We consider the following model equation for the Mackey-Glass oscillator [1]:

$$
\frac{d x}{d t}=-x+\frac{a x\left(t-\tau_{S}\right)}{1+x^{c}\left(t-\tau_{S}\right)},
$$

with $t$ being a dimensionless time, $\tau_{S}$ the time delay feedback, $a$ the feedback strength, and $c$ the degree of nonlinear- ity. In particular, we choose the typical values $a=2, c=10$, and $\tau_{S}=60$ for which the system operates in a chaotic regime. Time series were numerically integrated by using $\mathrm{He}$ un's method (also called the modified Euler's method) [41] with an integration step $\Delta t=0.01$ and sampling step $\delta t=0.2$ time units per sample. We analyzed time series with $N=10^{6}$ data points (the total integration time was $2 \times 10^{5}$ time units).

In Fig. 1 we plot the normalized permutation entropy $\mathcal{H}_{S}$ and the permutation $\mathrm{SCM} \mathcal{C}_{J S}$ as functions of the embedding delay $\tau$ for different embedding dimensions $(4 \leq D \leq 8)$. It can be clearly observed that these quantifiers have sharp and well-defined minima and maxima, respectively, when the embedding delay $\tau$ of the symbolic reconstruction is very close to the intrinsic time delay $\tau_{S}$ of the system, i.e., for $\tau$ near $300\left(\tau_{S} / \delta t=300\right)$. These extrema are due to an enhancement of time correlations for this value of $\tau$, resulting in the probability distribution of the ordinal patterns being different from the uniform probability distribution. Consequently, the permutation entropy decreases and the permutation statistical complexity increases, revealing the presence of some degree of order. This behavior is the hallmark of time delayed dynamics. It is interesting to note that in cases in which the system has no delayed dynamics, the quantifiers do not develop clear extrema that could generate spurious delay identification. We have analyzed the behaviors of the permutation entropy and permutation statistical complexity as functions of the embedding delay $\tau$ for numerical simulations of the different coordinates of the Lorenz system [42] in the chaotic regime, and we have not found pronounced extrema.

From Figs. 1(b) and 1(c) it can be concluded a slight time delay overestimation. This overestimation can be attributed to the internal response time or inertia of the Mackey-Glass system. The inertia is an inherent property difficult to determine precisely and affects most of the methods proposed to identify time delay from time series $[15,43,44]$. We have estimated the same time delay by using the autocorrelation function and the DMI since the inertia also affects these conventional techniques. It can also be seen from Figs. 1(b) and 1(c) that the time delay estimation is independent of the embedding dimension value. The best discrimination is obtained for the largest allowed value of $D$. By increasing the length and the number of symbols, i.e., by increasing the embedding dimension $D$, more information is being included when estimating any quantifier. Thus, it is reasonable that a better detection can be achieved with higher embedding dimensions. It is worth noting that there are other minima and maxima for the permutation entropy and permutation statistical complexity, respectively, but being less pronounced. These other peaks correspond to harmonics and subharmon$i c s$ of the system's time delay $\tau_{S}$. Thus, their presence contributes to the identification of the time delay. In the case of the permutation statistical complexity, the amplitude of the peak associated with the delay of the system has the largest amplitude, as can be seen in Fig. 1(a). We attribute this particular behavior to a reinforcement of the system delay effect associated with the special way of choosing the delay embedding sequence. According to the results shown in Fig. 1(a), we conclude that the permutation statistical complexity identifies the system delay better than the permutation entropy because the contrast with the base line is higher. It was 

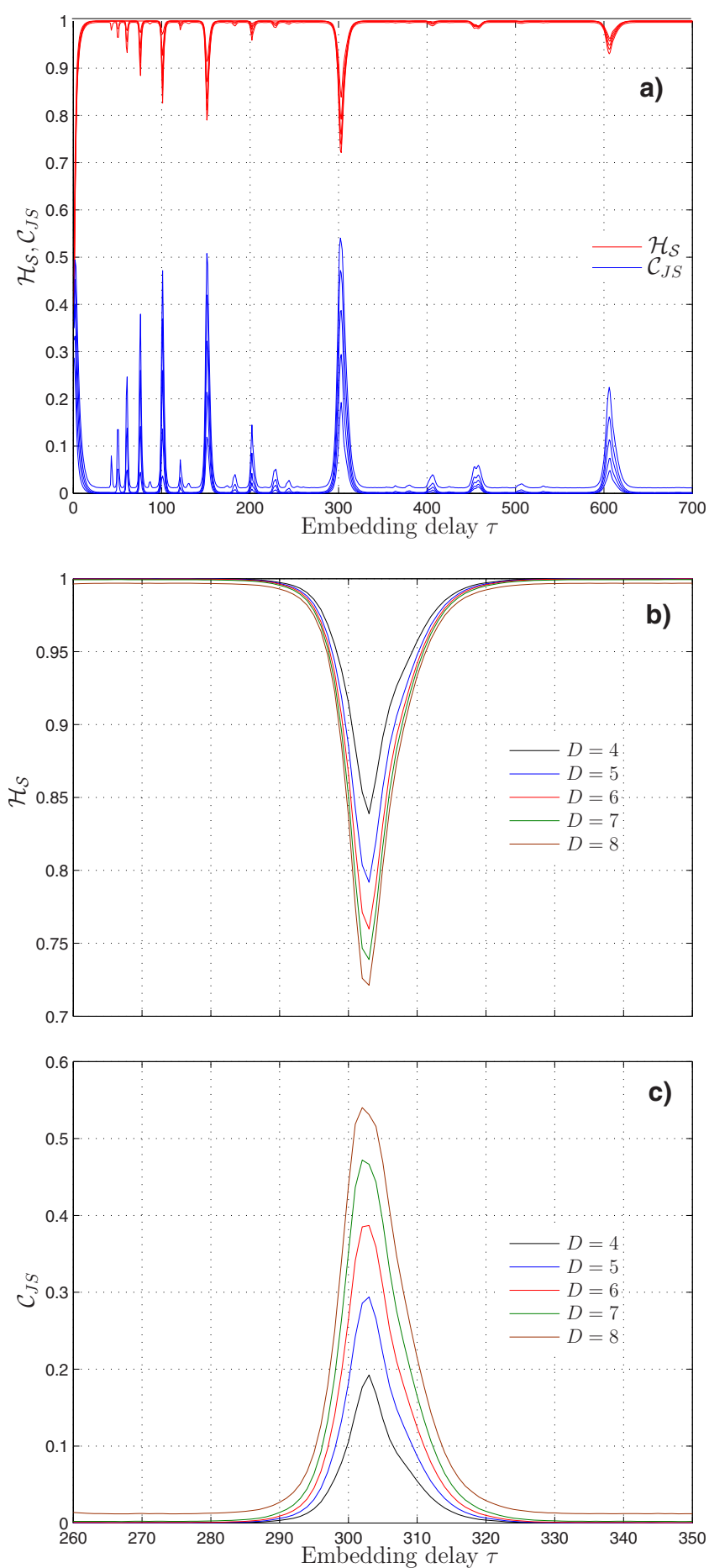

FIG. 1. (Color online) (a) Permutation entropy $\mathcal{H}_{S}$ and permutation statistical complexity $\mathcal{C}_{J S}$ as a function of the embedding delay $\tau$ for embedding dimensions $4 \leq D \leq 8$ ( $N=10^{6}$ data points). Enlargement near the time delay $\tau_{S}$ of the system in order to see more clearly the effect of the embedding dimension on the (b) $\mathcal{H}_{S}$ and (c) $\mathcal{C}_{J S}$ estimations. $D$ increases from top to bottom for $\mathcal{H}_{S}$ and from bottom to top for $\mathcal{C}_{J S}$.

recently shown that, in some cases $[39,45,46]$, the statistical complexity can be a particularly useful and efficient information theoretical quantifier. Based on these previous conclusions, from now on, we continue the analysis by considering

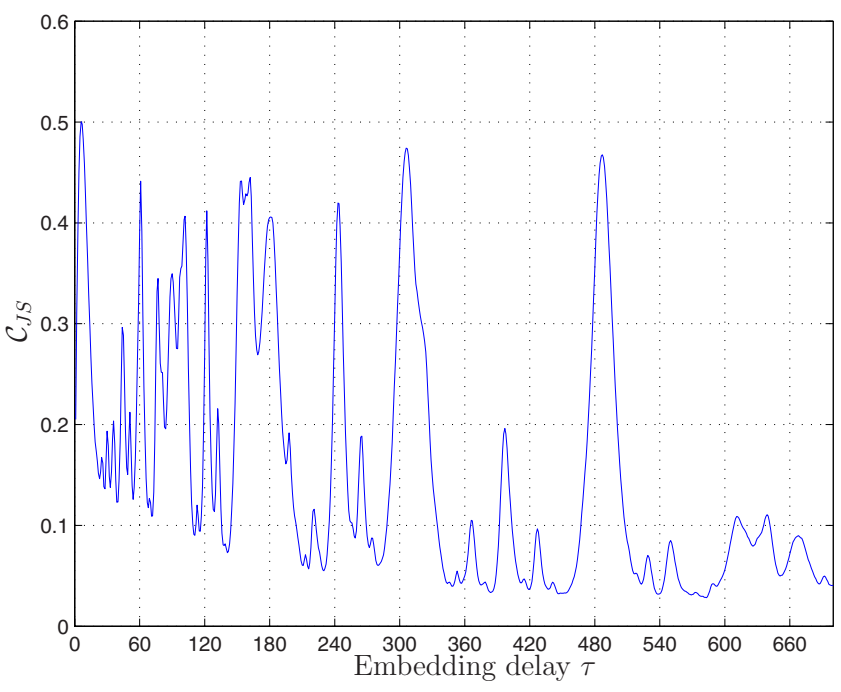

FIG. 2. (Color online) Permutation statistical complexity $\mathcal{C}_{J S}$ as a function of the embedding delay $\tau$ for embedding dimensions $D$ $=8\left(N=10^{6}\right.$ data points $)$ in the case of a Mackey-Glass system with time delays $\tau_{S, 1}=60$ and $\tau_{S, 2}=96$.

that the permutation statistical complexity $\mathcal{C}_{J S}$ with embedding dimension $D=8$ is the best quantifier to reach the goal of identifying the system's time delay under study.

To go further we analyzed the case of two time delays. Numerical data were obtained extending Eq. (5) to include two time delays by employing the same generalization followed in Ref. [47],

$$
\frac{d x}{d t}=-x+\frac{1}{2} \sum_{i=1}^{2} \frac{a x\left(t-\tau_{S, i}\right)}{1+x^{c}\left(t-\tau_{S, i}\right)} .
$$

The same parameters $(a=2, c=10)$ and integration method as in the one time delay case were implemented. Figure 2

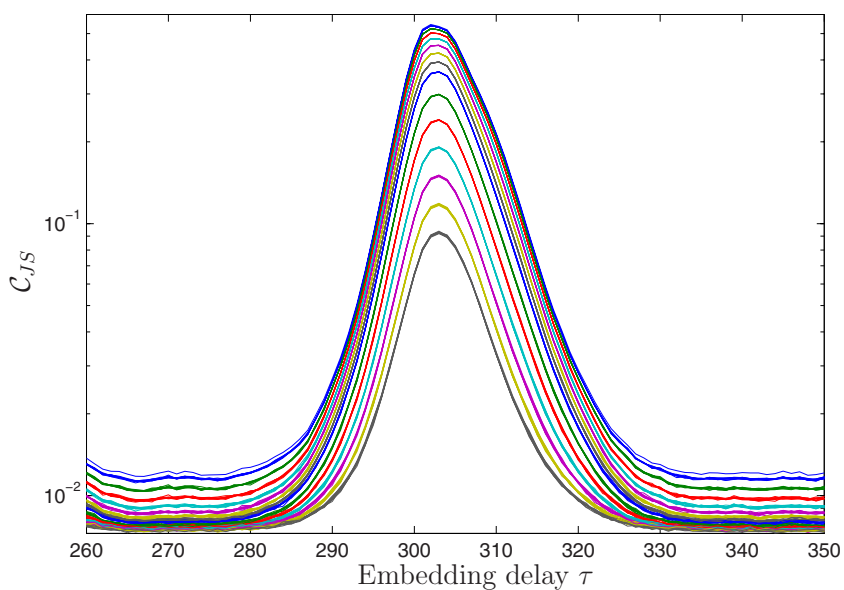

FIG. 3. (Color online) Semilogarithmic plot of the permutation statistical complexity $\mathcal{C}_{J S}$ as a function of the embedding delay $\tau$ for different levels of observational noise. The noise level associated with the different curves ( $\mathrm{NL}=0.05,0.1,0.15,0.2,0.25,0.3$, $0.35,0.4,0.5,0.6,0.7,0.8,0.9,1.0)$ increases from top to bottom. The embedding dimension $D=8$ and $N=10^{6}$ data points. Ten independent realizations for each noise level are plotted. Since the dispersion is very small, the differences between these ten lines are hardly distinguishable. 


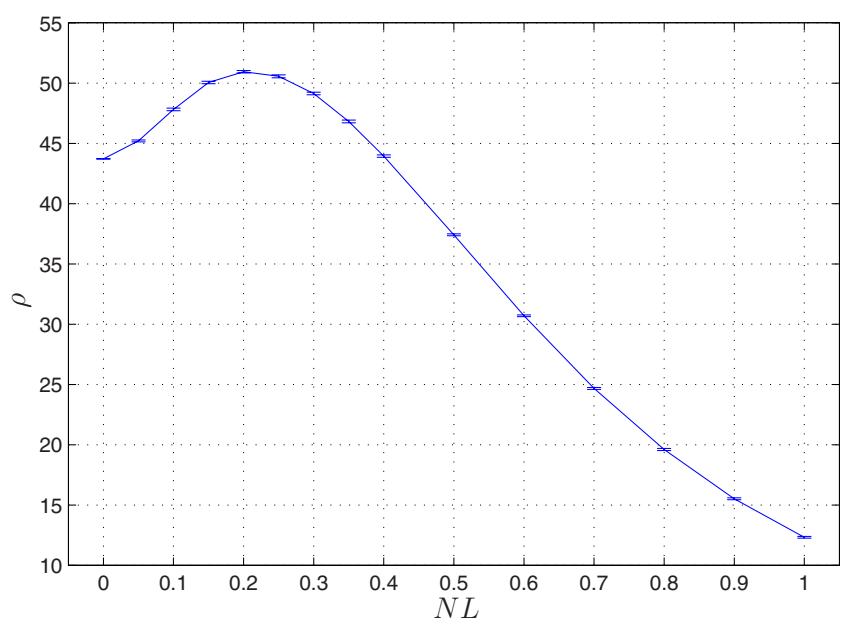

FIG. 4. (Color online) Ratio $\rho=\mathcal{C}_{J S}^{\text {peak }} / \mathcal{C}_{J S}^{\text {back }}$ as a function of the observational noise level. The embedding dimension was fixed equal to 8 and $N=10^{6}$ data points. Notice that the maximum ratio is obtained for a value of NL close to 0.2 and that more reliable time delay identifications are obtained with added observational noise in the range $0<\mathrm{NL}<0.4$. Error bars indicate standard deviations from ten independent realizations.

shows the behavior of the permutation statistical complexity $\mathcal{C}_{J S}$ as a function of the embedding delay $\tau$ for embedding dimensions $D=8$ in the case of a Mackey-Glass system with time delays $\tau_{S, 1}=60$ and $\tau_{S, 2}=96$. Pronounced maxima of the permutation statistical complexity for $\tau \sim 300\left(\tau_{S, 1} / \delta t=300\right)$ and $\tau \sim 480\left(\tau_{S, 2} / \delta t=480\right)$ allow us to identify the two time delays present in the system. Thus, multiple delays can also be identified with this methodology. The correct identification of multiple delays which are at commensurate ratios is in general a more complicated problem and will be addressed elsewhere.

Our next goal is to quantify the effect an observational additive noise has on the proposed approach. Since experi-

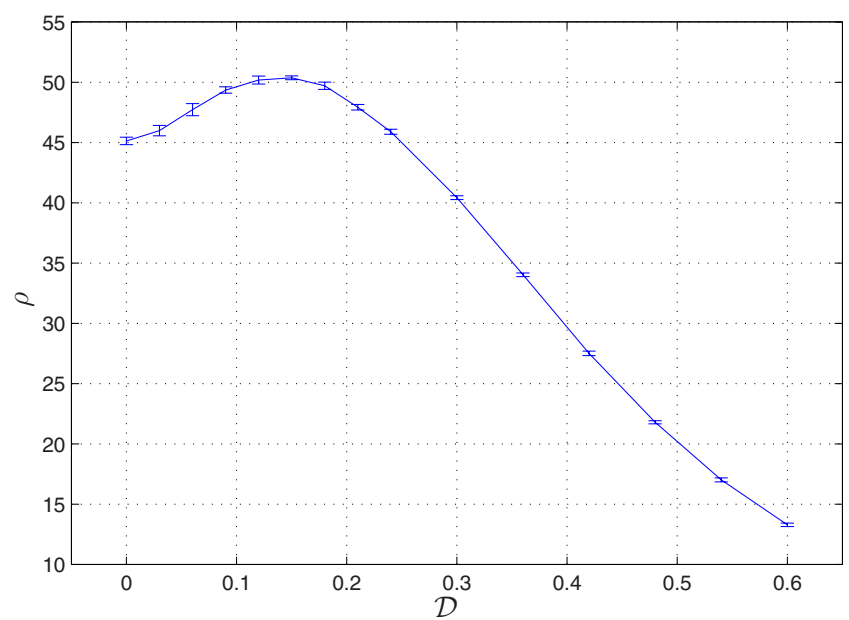

FIG. 5. (Color online) Ratio $\rho=\mathcal{C}_{J S}^{\text {peak }} / \mathcal{C}_{J S}^{\text {back }}$ as a function of the dynamical noise level. The embedding dimension was fixed equal to 8 and $N=10^{6}$ data points. Observe the maximum obtained for a Langevin force $\mathcal{D}$ of intensity near 0.15 . Error bars indicate standard deviations from ten independent realizations.
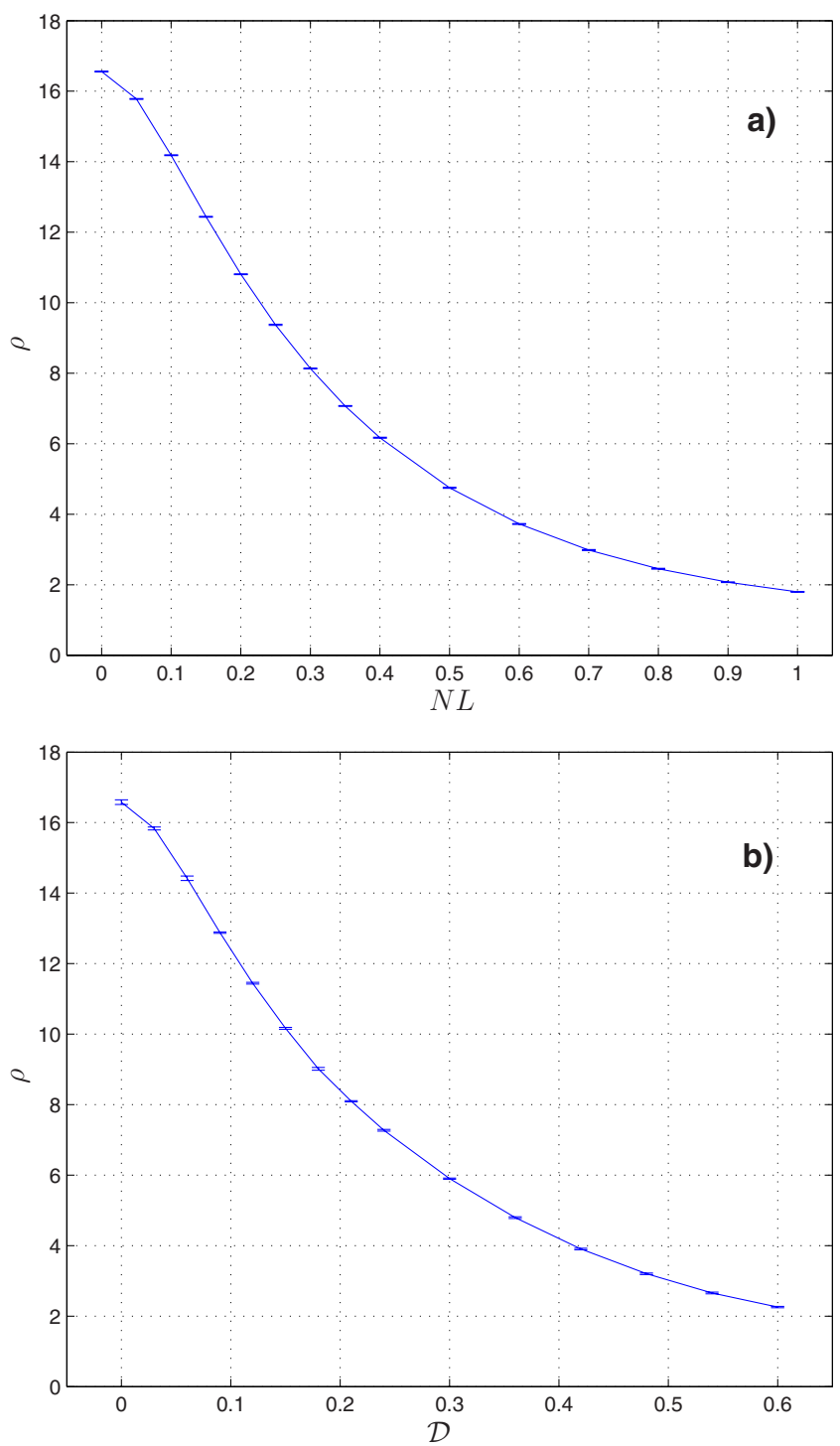

FIG. 6. (Color online) Signal-to-noise ratio $(\rho)$ for the delayed mutual information (DMI) as a function of (a) the observational noise level $(\mathrm{NL})$ and $(\mathrm{b})$ the dynamical noise level $(\mathcal{D})$. Error bars indicate standard deviations from ten independent realizations. The probability distributions were estimated with the usual histogram by using $2^{8}$ bins.

mental time series are naturally affected by a certain amount of observational noise, it is important to check the performance of our approach in the case of noisy time series. For this purpose a Gaussian white noise was added to the original Mackey-Glass simulated time series. Different noise levels (NL) from 0.05 to 1 , defined by the standard deviation of the noise divided by the standard deviation of the original signal, were considered. Ten independent realizations were taken into account in order to have better statistics. Figure 3 shows the performance of $\mathcal{C}_{J S}$ for $D=8$ in the region of interest, that is, around $\tau=300$. It can be clearly seen that our approach is very robust under the noise influence.

In order to better measure this effect, we have estimated the ratio between the amplitude at the delay feedback peak and the mean value of the background (the usual signal-tonoise ratio). The results are shown in Fig. 4. The resulting 

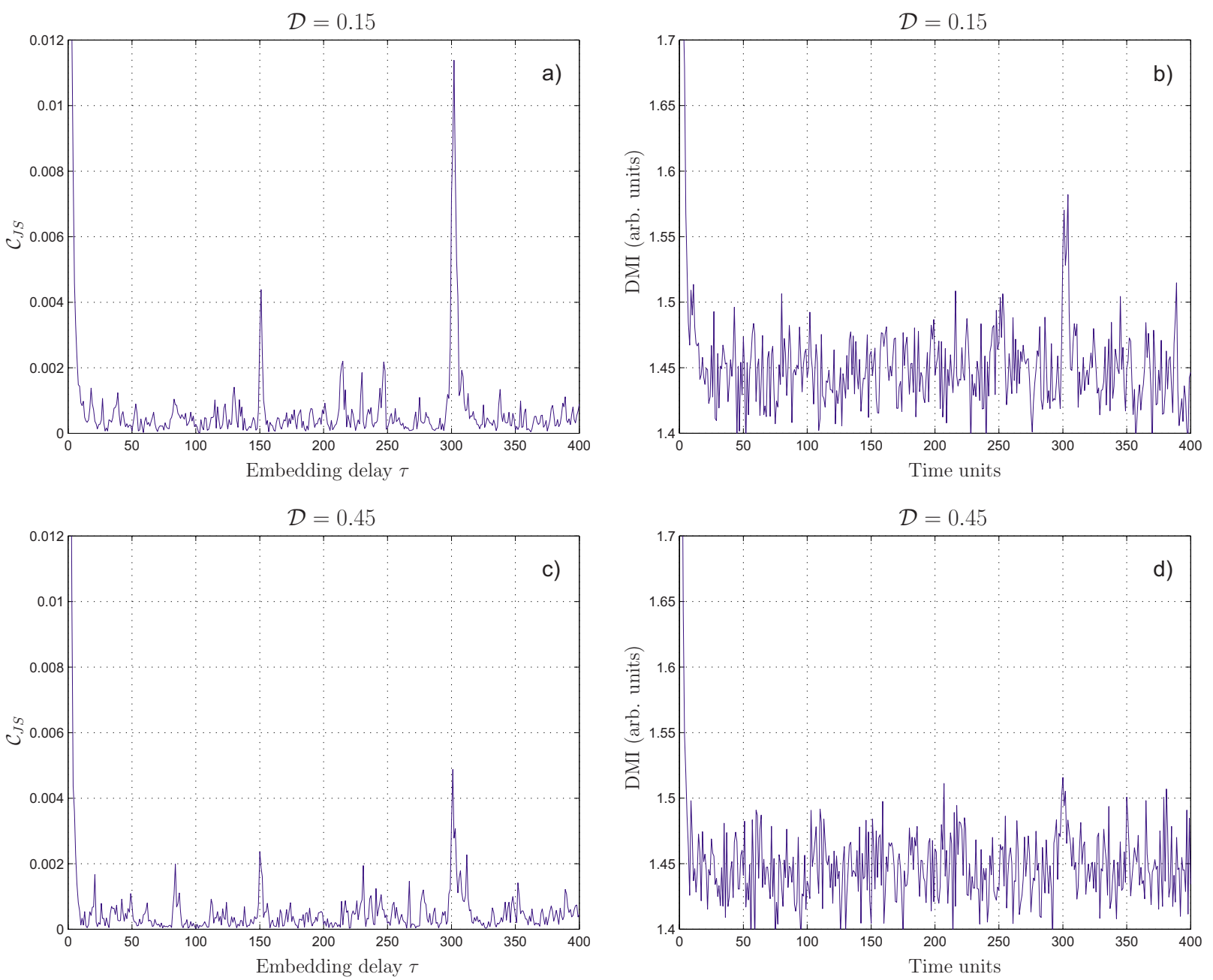

FIG. 7. (Color online) Comparison between the permutation statistical complexity $\left(\mathcal{C}_{J S}\right)$ and the DMI for the Mackey-Glass oscillator with low feedback strength $(a=1.2)$ and different dynamical noise levels: (a) and (b) $\mathcal{D}=0.15$, and (c) and (d) $\mathcal{D}=0.45$. The embedding dimension was fixed equal to 3 and $N=5 \times 10^{3}$ data points. DMI was estimated by using a histogram with $2^{8}$ bins.

plot displays a clear maximum of the ratio $\rho=\mathcal{C}_{I S}^{\text {peak }} / \mathcal{C}_{J S}^{\text {back }}$ at an intermediate noise level near 0.2. This value can be considered as the optimal amount of observational noise for the time delay identification purpose. It is worth noting that according to these results the identification of the time delay is more reliable in the presence of observational noise in the range $0<\mathrm{NL}<0.4$. A similar resonancelike behavior was recently found by Staniek and Lehnertz [25]. These authors analyzed the influence of a static (observational) noise in the detectability of directional coupling by estimating a symbolic transfer entropy. The ratio of the directionality indices for noisy and noise-free time series in a numerical example displays an analogous behavior (see, for instance, Fig. 3 of Ref. [25]). More importantly, the same symbolic technique, namely, the Bandt and Pompe permutation method, was adopted to estimate this quantifier.

With the aim of studying also the effect of a dynamical noise, we have simulated the Mackey-Glass system [Eq. (5)] including an additive Gaussian white noise term of zero mean and correlation $\mathcal{D}$. Langevin forces of different strengths $\mathcal{D}$ were considered. The results obtained for the ratio $\rho=\mathcal{C}_{J S}^{\text {peak }} / \mathcal{C}_{J S}^{\text {back }}$ as a function of different noise strengths are shown in Fig. 5. A resonancelike behavior is also observed which indicates a better performance of the quantifier in the presence of noise. A significant maximum for $\rho$ is found when $\mathcal{D}$ is near 0.15 .

We have also studied the effect that observational and dynamical noises have on the classical delayed mutual information in order to compare with the performance of our permutation-information-theory approach. In the case of the DMI the standard histogram was used to estimate the probability distribution associated with the time series. In particular, $2^{8}$ nonoverlapping equal-sized consecutive subintervals were employed to divide the full range. Similar results were obtained with other numbers of bins. From Fig. 6 it can be concluded that the performance of this information theory standard approach gets worse in the presence of noise. The signal-to-noise ratio is a continuously decreasing function of the noise level for both observational and dynamical noises. Thus, we stress the fact that our permutation information approach is particularly useful and efficient for experimental data where noise is inherently present.

It is well known that the time delay identification becomes more difficult for smaller values of the feedback 

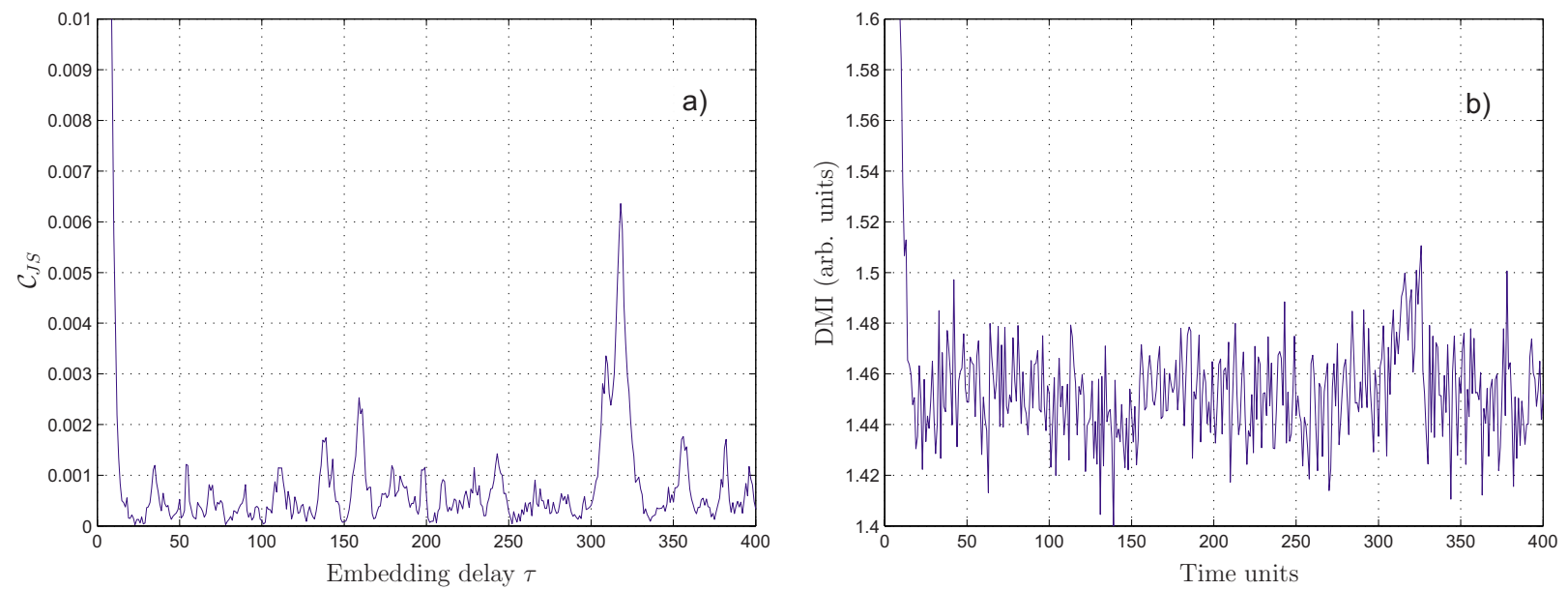

FIG. 8. (Color online) Time delay identification for a ring of four unidirectionally delay-coupled Mackey-Glass oscillators in the presence of dynamical noise $(\mathcal{D}=0.1)$. (a) Permutation statistical complexity $\left(\mathcal{C}_{J S}\right)$ with embedding dimension $D=3$ and (b) DMI estimated by using a histogram with $2^{8}$ bins. For this realization both quantifiers are able to identify the presence of the feedback delay at 300 time units.

strength or stronger nonlinearity. Rontani et al. [43,44] showed that the time delay signature of a chaotic semiconductor laser with optical feedback can be blurred when the feedback rate is relatively weak. With the aim of checking the ability of the proposed approach in this more severe time delay identification scenario, we have analyzed numerical simulations of the Mackey-Glass system with the same parameters $\left(c=10\right.$ and $\left.\tau_{S}=60\right)$ but low feedback strength ( $a$ $=1.2$ ). Moreover, additive Gaussian white noises of different intensities were added. We have also reduced the length of simulations to $N=5 \times 10^{3}$ data points. Figure 7 compares the results obtained for the permutation statistical complexity and the delayed mutual information for two different noise strengths $\mathcal{D}=0.15$ and 0.45 . It can be concluded that the time delay is clearly recovered by our approach in both cases. However, the recovery of the time delay for DMI is less straightforward. Particularly, in the noisier scenario $(\mathcal{D}$ $=0.45$ ) the time delay peak is on the order of the fluctuating background.

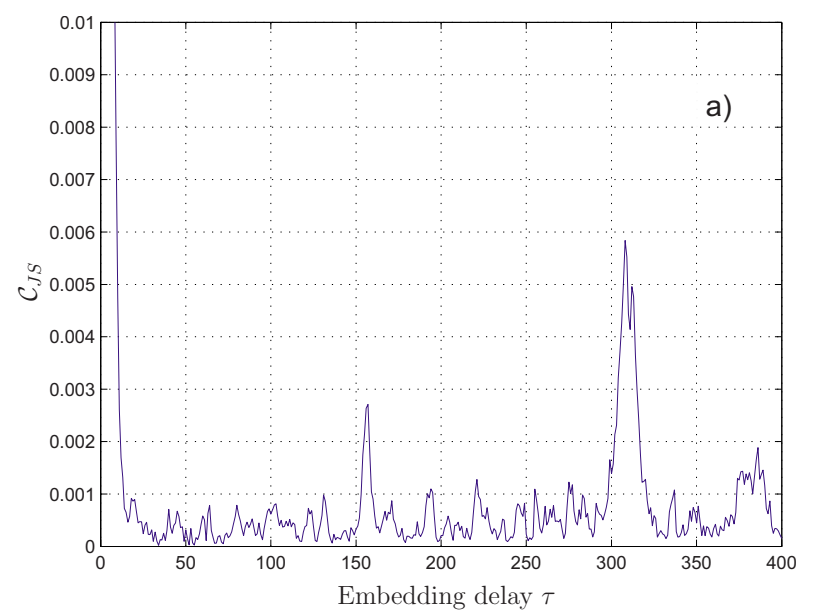

Finally, in order to test the performance of the permutation statistical complexity quantifier in a system with high nonlinearity, we analyze numerical simulations of a ring of four unidirectionally delay-coupled Mackey-Glass oscillators in the presence of dynamical noise. It has been recently reported that the fingerprint of the time delay can be significantly reduced in this system due to the stronger nonlinearity [48]. 100 independent numerical realizations of length $N$ $=2^{13}$ data points with additive Gaussian white noise of intensity $\mathcal{D}=0.1$ were considered for this system. We find that our permutation approach is able to discriminate the presence of the time delay in about $50 \%$ of cases and the DMI is successful in only $25 \%$ of realizations. For illustrating the results obtained we show in Fig. 8 a particular realization in which both quantifiers are successful and, in Fig. 9, another realization in which only the permutation quantifier is able to unveil the time delay. It is worth mentioning that our permutation approach is successful in all realizations where the DMI gives a positive answer.

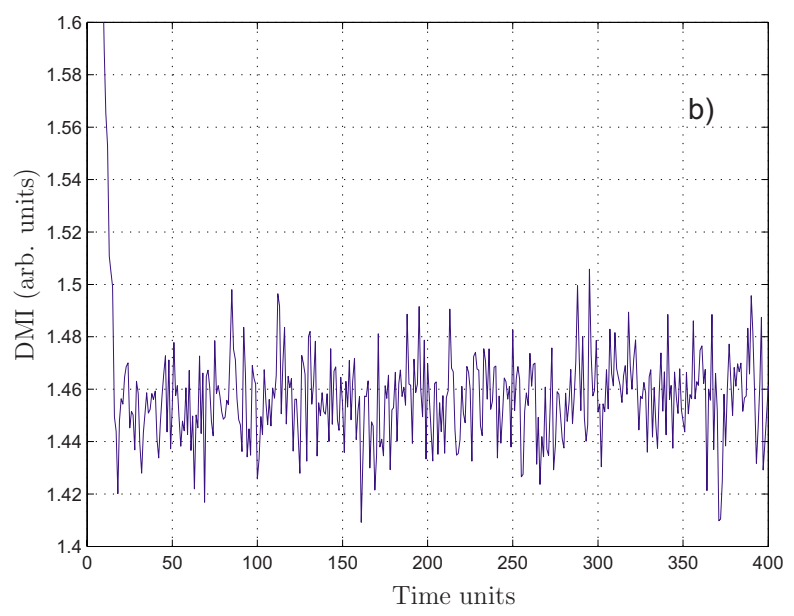

FIG. 9. (Color online) Time delay identification for a ring of four unidirectionally delay-coupled Mackey-Glass oscillators in the presence of dynamical noise $(\mathcal{D}=0.1)$. (a) Permutation statistical complexity $\left(\mathcal{C}_{J S}\right)$ with embedding dimension $D=3$ and (b) DMI estimated by using a histogram with $2^{8}$ bins. For this realization only the permutation quantifier is able to identify the presence of the feedback delay at 300 time units. 


\section{CONCLUSIONS}

Delay phenomena are of considerable practical importance. Thus, time delay identification from experimental time series within an inherent noise environment is, nowadays, an important challenge. In this work we introduced a reliable and simple approach to perform this task. Two different information theory quantifiers estimated by using an efficient symbolic technique, namely, the permutation entropy and the permutation statistical complexity, are able to reveal the presence of a time delay in the standard well-known Mackey-Glass system. The fingerprint of the time delayed dynamics is associated with a minimum of the permutation entropy and, simultaneously, a maximum of the permutation statistical complexity. Moreover, it has been shown that the latter quantifier is more sensitive for the time delay identification purpose than the entropy quantifier. By analyzing the influence of additive observational and dynamical noises we found a noise-enhanced phenomenon: the time delay identification can be improved by the presence of noise. This result is particularly valuable for analyzing experimental data. We have also shown that our permutation approach is useful for unveiling the presence of a time delay in difficult time delay identification scenarios, such as systems with low feedback rates or strong nonlinearities, even if only small data sets are available. A more in depth analysis for gaining insights into the nature of the noise-enhanced mechanism together with real experimental testing will be the goal of a next study.

\section{ACKNOWLEDGMENTS}

We thank Dr. L. Pesquera for very useful discussions and comments on the current research. L.Z. and O.A.R. were supported by Consejo Nacional de Investigaciones Científicas y Técnicas (CONICET), Argentina. The work of M.C.S. was supported by MEC (Spain) under a "Juan de la Cierva" contract. O.A.R. is supported by PVE of CAPES, Brazil. Part of this work was funded by MEC (Spain), MICINN (Spain), and FEDER under Projects No. TEC2006-10009/MIC (PhoDeCC), No. TEC2009-14101 (DeCoDicA), and No. FIS2007-60327 (FISICOS), and by the EC Project PHOCUS, European Commission FET-Open Grant No. 240763.
[1] M. C. Mackey and L. Glass, Science 197, 287 (1977).

[2] R. M. May, J. R. Beddington, C. W. Clark, S. J. Holt, and R. M. La, Science 205, 267 (1979).

[3] W. S. C. Gurney, S. P. Blythe, and R. M. Nisbet, Nature (London) 287, 17 (1980).

[4] K. Ikeda, Opt. Commun. 30, 257 (1979).

[5] R. Lang and K. Kobayashi, IEEE J. Quantum Electron. 16, 347 (1980).

[6] K. Ikeda and K. Matsumoto, Physica D 29, 223 (1987).

[7] E. Tziperman, L. Stone, M. A. Cane, and H. Jarosh, Science 264, 72 (1994).

[8] S. Lepri, G. Giacomelli, A. Politi, and F. T. Arecchi, Physica D 70, 235 (1994).

[9] A. M. Fraser and H. L. Swinney, Phys. Rev. A 33, 1134 (1986).

[10] V. S. Udaltsov, J.-P. Goedgebuer, L. Larger, J.-B. Cuenot, P. Levy, and W. T. Rhodes, Phys. Lett. A 308, 54 (2003).

[11] A. C. Fowler and G. Kember, Phys. Lett. A 175, 402 (1993).

[12] R. Hegger, M. J. Bünner, H. Kantz, and A. Giaquinta, Phys. Rev. Lett. 81, 558 (1998).

[13] Y.-C. Tian and F. Gao, Physica D 108, 113 (1997).

[14] R. K. Azad, J. S. Rao, and R. Ramaswamy, Chaos, Solitons Fractals 14, 633 (2002).

[15] M. J. Bünner, A. Kittel, J. Parisi, I. Fischer, and W. Elsäßer, EPL 42, 353 (1998).

[16] B. P. Bezruchko, A. S. Karavaev, V. I. Ponomarenko, and M. D. Prokhorov, Phys. Rev. E 64, 056216 (2001).

[17] M. Siefert, Phys. Rev. E 76, 026215 (2007).

[18] C. Bandt and B. Pompe, Phys. Rev. Lett. 88, 174102 (2002).

[19] J. M. Finn, J. D. Goettee, Z. Toroczkai, M. Anghel, and B. P. Wood, Chaos 13, 444 (2003).

[20] E. M. Bollt, T. Stanford, Y.-C. Lai, and K. Życzkowski, Phys. Rev. Lett. 85, 3524 (2000).

[21] C. S. Daw, C. E. A. Finney, and E. R. Tracy, Rev. Sci. Instrum.
74, 915 (2003).

[22] Y. Cao, W. W. Tung, J. B. Gao, V. A. Protopopescu, and L. M. Hively, Phys. Rev. E 70, 046217 (2004).

[23] O. A. Rosso, H. A. Larrondo, M. T. Martín, A. Plastino, and M. A. Fuentes, Phys. Rev. Lett. 99, 154102 (2007).

[24] O. A. Rosso, L. Zunino, D. G. Pérez, A. Figliola, H. A. Larrondo, M. Garavaglia, M. T. Martín, and A. Plastino, Phys. Rev. E 76, 061114 (2007).

[25] M. Staniek and K. Lehnertz, Phys. Rev. Lett. 100, 158101 (2008).

[26] A. Bahraminasab, F. Ghasemi, A. Stefanovska, P. V. E. McClintock, and H. Kantz, Phys. Rev. Lett. 100, 084101 (2008).

[27] L. Zunino, D. G. Pérez, M. T. Martín, M. Garavaglia, A. Plastino, and O. A. Rosso, Phys. Lett. A 372, 4768 (2008).

[28] G. Ouyang, X. Li, C. Dang, and D. A. Richards, Phys. Rev. E 79, 041146 (2009).

[29] O. A. Rosso and C. Masoller, Phys. Rev. E 79, 040106(R) (2009).

[30] O. A. Rosso and C. Masoller, Eur. Phys. J. B 69, 37 (2009).

[31] R. Monetti, W. Bunk, T. Aschenbrenner, and F. Jamitzky, Phys. Rev. E 79, 046207 (2009).

[32] D. P. Feldman and J. P. Crutchfield, Phys. Lett. A 238, 244 (1998).

[33] D. P. Feldman, C. S. McTague, and J. P. Crutchfield, Chaos 18, 043106 (2008).

[34] P. W. Lamberti, M. T. Martín, A. Plastino, and O. A. Rosso, Physica A 334, 119 (2004).

[35] R. López-Ruiz, H. L. Mancini, and X. Calbet, Phys. Lett. A 209, 321 (1995).

[36] I. Grosse, P. Bernaola-Galván, P. Carpena, R. Román-Roldán, J. Oliver, and H. E. Stanley, Phys. Rev. E 65, 041905 (2002).

[37] X. Calbet and R. López-Ruiz, Phys. Rev. E 63, 066116 (2001).

[38] M. T. Martin, A. Plastino, and O. A. Rosso, Physica A 369, 439 (2006). 
[39] J. R. Sánchez and R. López-Ruiz, Physica A 355, 633 (2005).

[40] M. Staniek and K. Lehnertz, Int. J. Bifurcation Chaos 17, 3729 (2007).

[41] T. C. Gard, Introduction to Stochastic Differential Equations (Marcel Dekker, New York, 1988).

[42] S. H. Strogatz, Nonlinear Dynamics and Chaos: With Applications to Physics, Biology, Chemistry, and Engineering (Studies in Nonlinearity) (Westview Press, Cambridge, Massachusetts, 2001).

[43] D. Rontani, A. Locquet, M. Sciamanna, and D. S. Citrin, Opt.
Lett. 32, 2960 (2007).

[44] D. Rontani, A. Locquet, M. Sciamanna, D. S. Citrin, and S. Ortin, IEEE J. Quantum Electron. 45, 879 (2009).

[45] J. Sañudo and R. López-Ruiz, Phys. Lett. A 372, 5283 (2008).

[46] C. C. Moustakidis, V. P. Psonis, K. C. Chatzisavvas, C. P. Panos, and S. E. Massen, Phys. Rev. E 81, 011104 (2010).

[47] H. Voss and J. Kurths, Phys. Lett. A 234, 336 (1997).

[48] G. Van der Sande, M. C. Soriano, I. Fischer, and C. R. Mirasso, Phys. Rev. E 77, 055202(R) (2008). 